\title{
A New, Open and Modular Platform for Research in Autonomous Four-Legged Robots
}

\author{
Martin Friedmann ${ }^{1}$, Sebastian Petters ${ }^{1}$, Max Risler ${ }^{1}$, Hajime Sakamoto ${ }^{2}$, \\ Oskar von Stryk ${ }^{1}$, and Dirk Thomas ${ }^{1}$ \\ 1 Simulation, Systems Optimization and Robotics Group, Technische Universität \\ Darmstadt, Hochschulstr. 10, D-64289 Darmstadt, Germany \\ Web: www.sim.tu-darmstadt.de \\ ${ }^{2}$ Hajime Research Institue, Ltd., Osaka, Japan
}

\begin{abstract}
In this paper the design goals for a new, open and modular, four-legged robot platform are described that was developed in reaction to the open call for a standard platform issued by the RoboCup Federation in 2006. The new robot should have similar motion and sensing capabilities like the previously used Sony AIBO plus several new ones. The hardware and software should be open, modular and reconfigurable. The robot should be resonably priced and allow annually upgrades.
\end{abstract}

\section{Introduction}

Between 1999 when Sony released the first of in total three robot generations and January 2006 when Sony announced the discontinuation of its production the AIBO robot [1] had found remarkable acceptance. It was, however, not only used for its original determination as a home entertainment robot but also found widespread use as an affordable standard platform for many research and teaching projects in robotic agents at universities. This was a result of Sony's publication of a programmable interface based on OPEN-R [2] which allowed to read the sensors and to send commands to the actuators.

One of the many applications and presumable the most popular is the use and investigation of the robot as a standard platform for autonomous robot soccer teams in the Four-Legged Robot League [3] of the RoboCup [4] since 1999. One of the main features of this league is the fact that all teams use the same standard robot platform and, besides of wear and tear, only the software differs which allows to evaluate different approaches, e.g., for vision, behavior and motion, in a challenging benchmark scenario.

As a reaction to the discontinuation of the AIBO a call for tenders for a standard robot platform for robot soccer was announced by the RoboCup Federation in November 2006 [4] with the following characteristics:

- A standard platform: No hardware development should be required (or allowed) by the teams during competitions. The robot must have an operating system and software development environment allowing full control of sensors and actuators. The platform should be modular, permitting upgrades and modifications from year to year. 
- Many degrees of freedom: Quadruped robots allow for a large variety of gaits and kicks. The new platform need not be a quadruped but it should have a sufficient number of degrees of freedom that teams can create interesting new behaviours and modes of locomotion.

- Directed perception: The robot is required to have onboard colour vision but not an omnidirectional camera since it is wished that teams develop methods for active perception.

- Full Autonomy: The robots are required to be fully autonomous. That is, they must have on-board computing resources sufficient to meet all processing requirements. No off-board computation is allowed. The robots must operate with no intervention by team members and minimal intervention by referees. Games typically consist of two half parts, each of them lasting for around $15 \mathrm{~min}$, with a $10 \mathrm{mn}$ break.

- Wireless communication: The robots must be capable of wireless 802.11 communications permitting the exchange of information between robots on the field.

- Physical characteristics: Ideally, the robot should be relatively small such that several can fit comfortably on one of the current RoboCup fields. Robots that are visually appealing will be looked upon favourably.

- (optional) Compatibility with some available robot development software.

Among the eight submitted proposals four tenderers including the robot presented in this paper were invited to demonstrate their robots during RoboCup 2007 in July in Atlanta. This paper describes the design issues and selections undertaken to meet the above mentioned requirements in mechanics and kinematics, sensing and computing abilities for the proposed four-legged robot including a comparison with the currently used Sony AIBO ERS-7 robot in the four-legged robot league.

\section{Design of the Four Legged Robot}

The call for tenders would in principle also allow a humanoid robot. However, the authors which already have developed a powerful humanoid robot [5] refrained from this variant as the cost for such a single, powerful humanoid robot would result in an overall cost comparable to that for a whole team of four or five fourlegged robots. This is due because of the larger number of motors with much higher performance and cost needed for a powerful humanoid robot compared with a four-legged robot.

The proposed new four-legged robot not only features a modular, kit style design which facilitates the extension by further actuators or sensors but also facilitates repair and maintenance and, thus, reduces cost for purchase and operation of the robot. Furthermore, the new robot has similar motion and sensing capabilities as the Sony AIBO plus several additional ones. 


\subsection{Mechanical Design}

Various kinematical designs were considered and tested in a 3D kinematic motion and camera simulation (see Fig. 1). Each leg consists of three rotational joints. Special attention was also given to the design of the robot's head and neck joints which features three degrees of freedom allowing to look in any direction including backwards (through the legs) as well as a wide range of motions for ball manipulation. The final kinematic design allows a wide range of motion capabilities which include the well established low stance walking on the forearms currently common in the Four-Legged Robot League. This walking gait has the advantage that a lower height of the center of gravity above ground is more stable with respect to shocks. Also the robot can see a ball directly in front of it and can still see parts of the environment. This is has not been possible for an upright gait with the AIBO. The new robot therefore is designed to being able to position the head for looking forward at high and also low height above the ground. Furthermore, the robot can look underneath its torso to the back and see the world behind upside down. It can now also kick the ball with the head underneath the torso to the back.
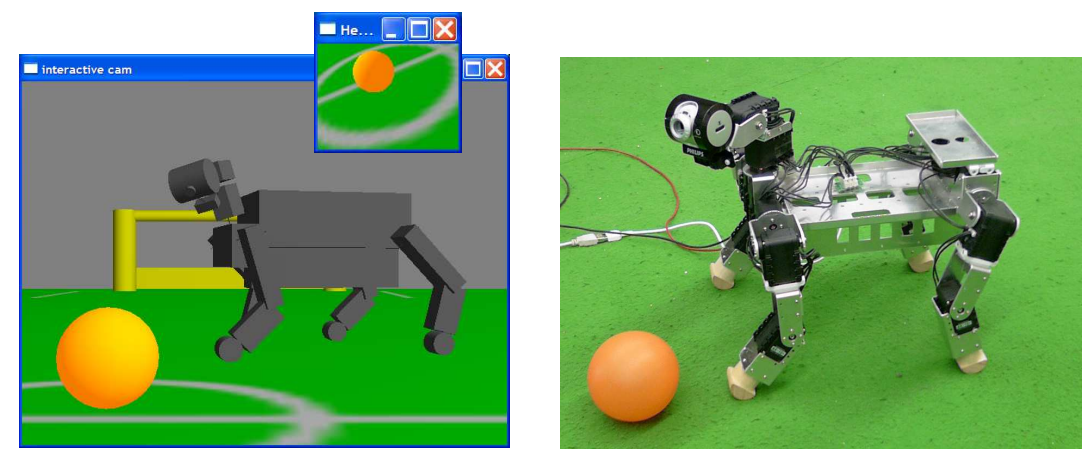

Fig. 1. Left: Simulation of the locomotion of the proposed four-legged robot and images captured from its simulated camera. Right: Robot model for the mechanical motion apparatus.

A servo motor module has been selected which provides a torque of $16.5 \mathrm{~kg} \mathrm{fcm}$ at $10 \mathrm{~V}$ operation voltage and speed of $0.196 / 60 \mathrm{degs}$ which is high enough to expect about the same speed of locomotion as can be achieved with a Sony AIBO in RoboCup. The servos are connected by a serial bus with the main controller, allowing high control rates $(125 \mathrm{~Hz}$ and higher). As the servos are linked by a daisy-chain bus, more servos can be added easily for research projects beyond the RoboCup. To provide constant conditions for the servos over the whole time of operation a switching voltage regulator is used. It provides a constant voltage independent from the charge status of the batteries. Furthermore, at the disposal of the robot programmer now are not only motor position but also temperature, load, input voltage and speed of the motors. 

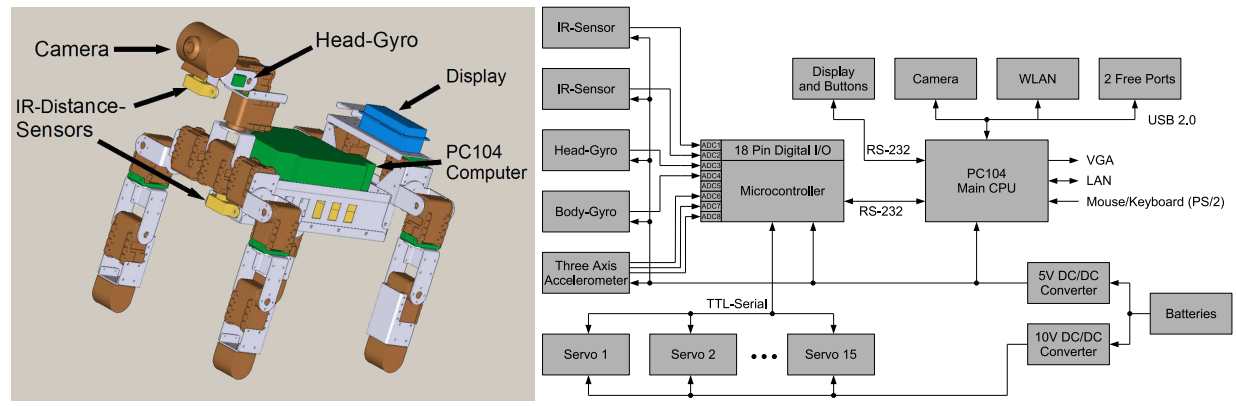

Fig. 2. Left: CAD-Drawing of the robot. Right: Structure of the robot's components.

\subsection{Sensing}

The main sensor of the robot is a Philips SNC900 webcam in the head. This camera provides a high resolution of $640 \times 480$ and frame rate of up to $90 \mathrm{fps}$. The horizontal aperture angle is 45 degrees. Drivers are available for current windows plattforms as well as for Linux. A driver for Windows CE as been developed as well.

Two infrared distance sensors in the chest and in the head, allow detection of objects independent of the motion of the robot's body. The selected sensor provides a measuring range of 10 to $80 \mathrm{~cm}$. In the torso of the robot, a 3D accelerometer is mounted to measure if the robot has fallen over and to which side. A 1D gyroscope measures the angular velocity around the vertical axis and can be used for improved odometry calculations. The use of an additional gyroscop in the head enables research in the field of inertial stabilization of the camera's field of view during walking. Further sensing capabilies are provided by the selected servo motors as mentioned earlier.

\subsection{Computing Capabilities}

The robot is equipped with an onboard $\mathrm{PC}$ and an additional microcontroller circuit for interfacing with the servos and sensors (see Fig. 2).

For the onboard PC a PC 104 standard platform with $500 \mathrm{MHz}$ was chosen, allowing easy exchange/upgrade of the CPU for projects with higher computational demands. The numerical coprocessor facilitates the development of algorithms with intensive floating-point arithmetics where as on the AIBO only fixed-point arithmetics was available. The selected computer has low battery requirements and does not need an external cooler. It includes USB 2.0, onboard graphics, sound interfaces and 100/1000 MBit Lan. Up to 1024MB of RAM may be installed. For wireless comunication an USB WLAN adapter will be included. On the robot's back a display (with two lines of 16 characters each) is provided enabling some online monitoring and debugging as well as 4 push buttons for programmable commands like start or stop. Both are accessed by RS232. 
On the backside of the robot is an opening which enables easy access to a number of I/O interfaces and to the LiPo batteries: a 1 GBit LAN Ethernet slot, a compact flash card slot, a plug-in for a monitor cable, and 2 free USB ports. The latter can be used very flexibly, e.g., to plug keyboard and mouse directly to the onboard computer or to add additional devices like a microphone, a loudspeaker or an extra camera.

The microcontroller circuit consists of a Renesas SH2/7125 MCU (MicroController-Unit) with $8 \mathrm{k}$ of flash-ROM and $4 \mathrm{k}$ of RAM. A control cycle time of $8 \mathrm{~ms}$ or shorter can be realized for the 15 motors. The MPU provides a wide range of $\mathrm{I} / \mathrm{O}$ capabilities including 8 analogue-digital-converters (ADC). Two ADCs are hard-wired to the most relevant directions of the acceleration sensor, the other six ADCs may be accessed freely by pin headers. In the circuit 18 digital I/O pins and may be accessed by pin headers. The firmware of the microcontroller will be open and a flash development kit is available which enables the robot programmer to modify the firmware as needed, e.g., for inverse kinematics calculations.

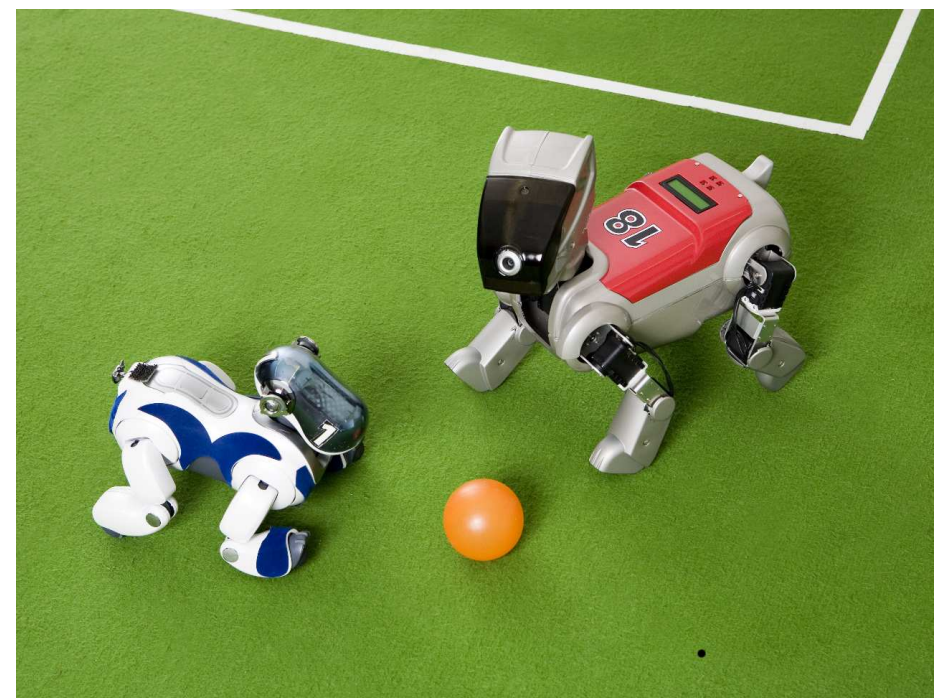

Fig. 3. The new four-legged robot on the right together with the robot previously used in the RoboCup Four-Legged Robot League (c) Katrin Binner/TU Darmstadt).

\subsection{Comparison with the Sony AIBO ERS-7}

The basic kinematics of the robot is roughly comparable to that of an AIBO with three degrees of freedom in each leg and in the head. The robot is expected being able to reach a similar fast walking speed. A similiar variety of gaits can 
be realized. Additionally, fast upright gaits can be implemented, which were not useful for the AIBO because of the design of its point-like feet-ground contact which provides only low friction foot contact in upright positions and leads to slipping of the feet when the legs propell the robot forward in upright walking position. The foot design of this robot provides enough friction at ground contact to develop fast upright walking gaits. Furthermore, the wider stance of the robot provides more robustness against collisions from the side and allows new ways of ball manipulation, e.g., kicking the ball behind the robot underneath its torso.

The camera resolution and image quality of $640 \times 480$ pixels is significantly larger than of the $208 \times 160$ pixels camera of the AIBO ERS-7. The new camera offers a significantly higher framerate of 90 instead of 30fps. The robot can reach about the same camera positions as the AIBO. But also additional positions are possible like looking to the front in high or low neck position above ground or looking back underneath the robot to see the world behind upside down.

As inertial sensors, the AIBO had one three-axis accelerometer in the torso whereas the new robot has in addition a $1 \mathrm{D}$ gyroscope in the head (to enable research in inertial stabilization of the field of view) as well as in the torso to measure angular rotation around the vertical axis (to be used for improved odometry calculations). Unlike AIBO the robot's servos offer the possibility to directly access temperature, load and torque values.

The proposed four-legged robot has a modular, kit style design. The robot programmers will have the possibility to select their preferred real-time operating system, e.g., Windows CE or RT-Linux or to reprogram the microcontroller. Both operating system will come with a basic software development kit and demo programs for basic robot operation. Despite the fact, that the robot is a verstaile platform for research in autonomous four-legged robots, most parts of the robot can easily be reused or reconfigured for other robot applications like the motors, the microcontroller, the camera, the main computer or the sensors.

\section{Conclusions and Outlook}

Design considerations and specifications for a new standard four-legged robot platform for research and education have been presented which offers many new research opportunities compared to the robot previously used in the Four-Legged Robot League of the RoboCup. Also careful considerations have been made to provide a platform useful for research beyond the field of robot soccer.

So far the motion capabilities of a first demonstrator robot have been tested and the complete computing and sensing hardware has been integrated into the robot. Also an outer design for the new four-legged robot has been developed in cooperation with Gotha Design, Germany (Fig. 3). Furthermore a first omnidirectional walking motion for remote control using a joystick has been implemented based on the the robot framework RoboFrame [6]. A demonstration to the international scientific public has been carried out successfully on July 7 during RoboCup 2007. 
The described robot provides an open, modular and reconfigurable platform for research in legged autonomous systems. As the system uses standard busses, it can be extended easily, e.g. by adding PC104 components. The main CPU board can be exchanged for other, stronger CPUs. Additional analogue and digital sensors may be added to the controller's spare ADC and digital I/O pins. Due to the serial bus additional servos can be added without changes to the hardware. The proposed new robot enables a large variety of motion and sensing capabilities and an open, modular and reconfigurable hardware and software design at a moderate price. The modular design also facilitates repair and maintenance. It is therefore well suited as a autonomous legged robot platform for universitary research and education.

More information about the robot including videos and photos is available from the website www.thenewrobot.com .

\section{References}

1. M. Fujita and H. Kitano. Development of an autonomous quadruped robot for robot entertainment. Autonomous Robots, 5(1):7-18, 1998.

2. M. Fujita and K. Kageyama. An open architecture for robot entertainment. In Proc. 1st Intl. Conf. on Autonomous Agents, pages 435 - 442, Marina del Rey, CA, USA, 1997.

3. M. Veloso, W. Uther, M. Fijita, M. Asada, and H. Kitano. Playing soccer with legged robots. In Proc. IEEE/RSJ Intl. Conf. on Intelligent Robots and Systems, pages 437-442, Victoria, BC, CAN, Oct. 13-17 1998.

4. RoboCup. www.robocup.org. webpage, RoboCup Federation.

5. M. Friedmann, J. Kiener, S. Petters, H. Sakamoto, D. Thomas, and O. von Stryk. Versatile, high-quality motions and behavior control of humanoid soccer robots. In Proc. Workshop on Humanoid Soccer Robots of the 2006 IEEE-RAS Int. Conf. on Humanoid Robots, pages 9-16, Genoa, Italy, Dec. 4-6 2006.

6. M. Friedmann, J. Kiener, S. Petters, D. Thomas, and O. von Stryk. Modular software architecture for teams of cooperating, heterogeneous robots. In Proc. IEEE Intl. Conf. on Robotics \& Biomimetics, pages 613-618, Kunming, China, Dec. 17-20 2006. 